\title{
Random allocation software for parallel group randomized trials Mahmood Saghaei*
}

\author{
Address: Department of Anaesthesia, Isfahan University of Medical Sciences, Isfahan, Iran \\ Email: Mahmood Saghaei* - saghaei@med.mui.ac.ir \\ * Corresponding author
}

Published: 09 November 2004

BMC Medical Research Methodology 2004, 4:26 doi:10.1 186/147|-2288-4-26

This article is available from: http://www.biomedcentral.com/I47I-2288/4/26

(C) 2004 Saghaei; licensee BioMed Central Ltd.

This is an Open Access article distributed under the terms of the Creative Commons Attribution License (http://creativecommons.org/licenses/by/2.0), which permits unrestricted use, distribution, and reproduction in any medium, provided the original work is properly cited.
Received: 17 August 2004

Accepted: 09 November 2004

\begin{abstract}
Background: Typically, randomization software should allow users to exert control over the different aspects of randomization including block design, provision of unique identifiers and control over the format and type of program output. While some of these characteristics have been addressed by available software, none of them have all of these capabilities integrated into one package. The main objective of the Random Allocation Software project was to enhance the user's control over different aspects of randomization in parallel group trials, including output type and format, structure and ordering of generated unique identifiers and enabling users to specify group names for more than two groups.
\end{abstract}

Results: The program has different settings for: simple and blocked randomizations; length, format and ordering of generated unique identifiers; type and format of program output; and saving sessions for future use. A formatted random list generated by this program can be used directly (without further formatting) by the coordinator of the research team to prepare and encode different drugs or instruments necessary for the parallel group trial.

Conclusions: Random Allocation Software enables users to control different attributes of the random allocation sequence and produce qualified lists for parallel group trials.

\section{Background}

An important aspect of any trial that should be clearly stated in the final report is the method used to assign treatments (or other interventions) to participants [1]. In the final report of the trial, authors should specify the method of sequence generation, i.e. whether they have used mechanical means, a computer generated random list or random number table. After preparing a random sequence, subjects will be allocated to the trial groups using an implementation method such as numbered containers, central telephone line, or allocation by a person who is not involved in the main research and patient care (the encoder). During the process of allocation each sub- ject will be given a unique identification code (Unique Identifier, UI). This UI will be used as a label to uniquely identify the patient's group after completion of the study. During the study period this UI will be given to the main researchers together with the necessary treatment (e.g. drug or placebo). Usually these treatments are prepared by the encoder with the same physical characteristics (shape, color, size, etc), differing only by the UI labels to blind the main researchers about the actual patient group. Following enrollment of all subjects into the study, these UIs are decoded to determine the patient group. Depending on the preference of the researchers or facilities of the research environment, subjects are randomly allocated to intervention groups using either a random list prepared 


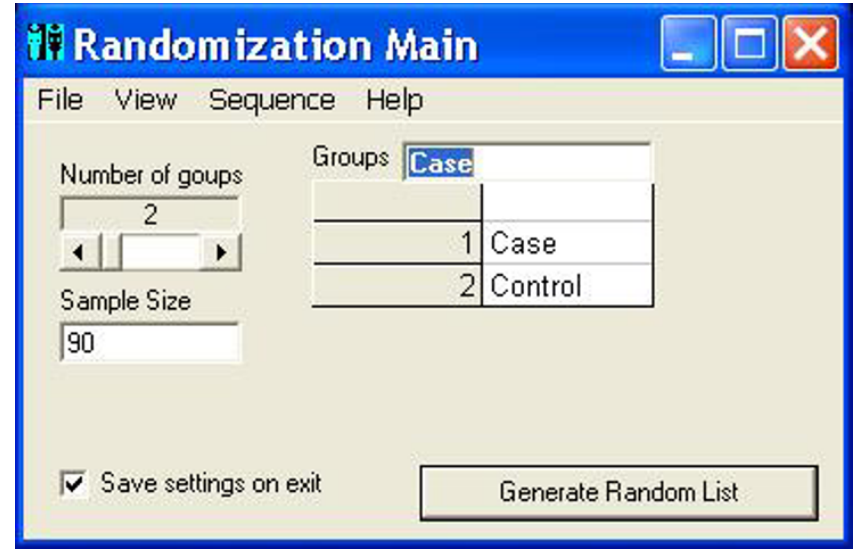

Figure I

Main window. The main window showing different options for number of groups, sample size, and group names.

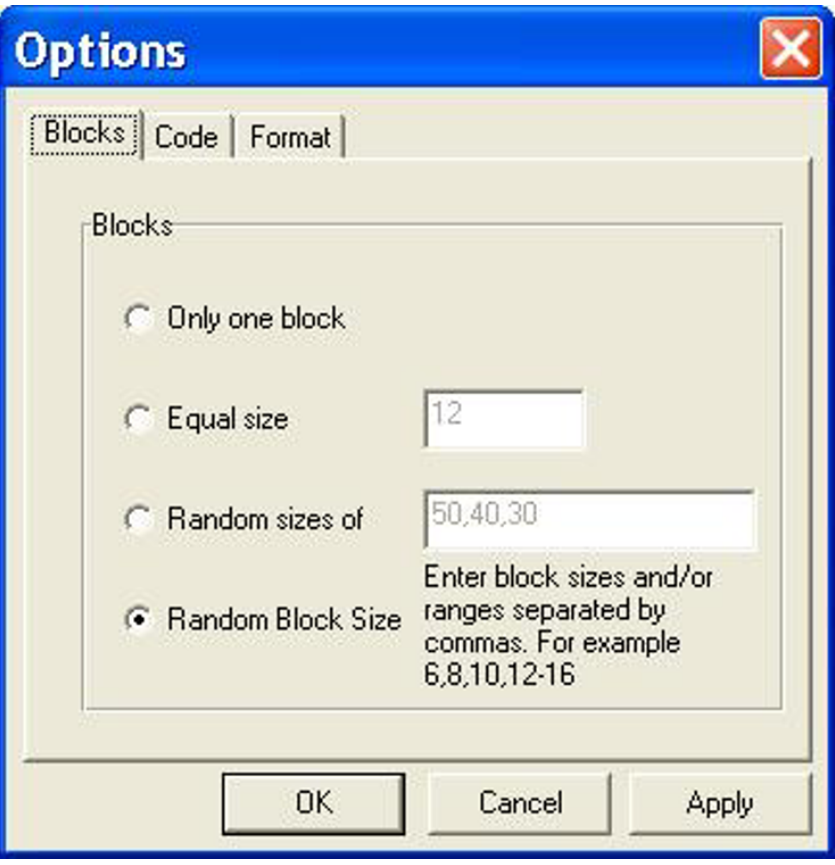

Figure 2

Options window: Blocks. Options window, settings for block design.

before the study (In Advance method) or a randomized allocation at the moment of intended intervention (Just In Time method; JIT). Both the JIT and In Advance meth-

\section{Options}

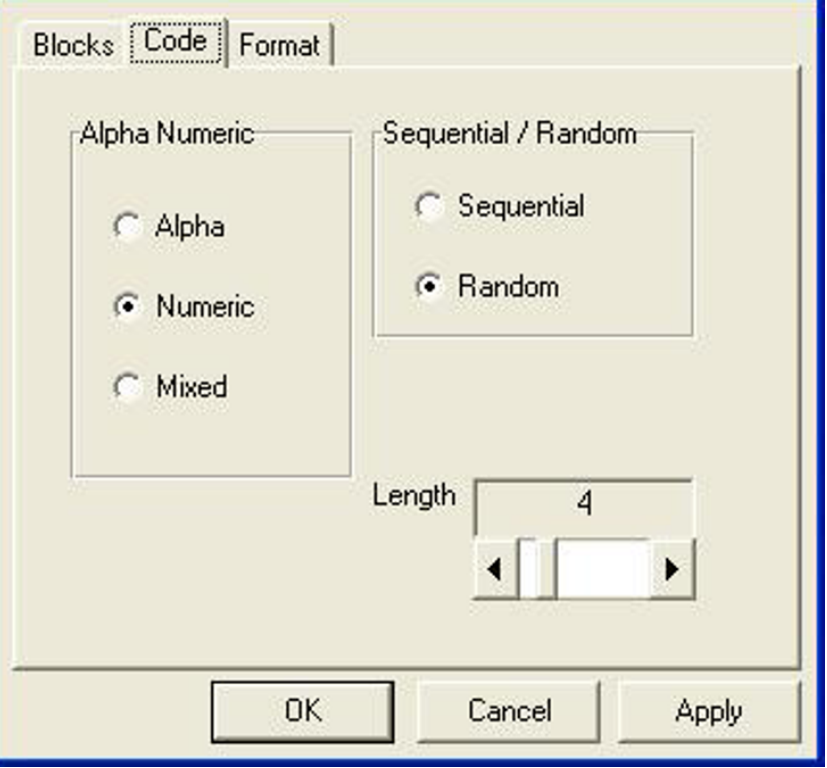

Figure 3

Options window: Code. Options window, setting the format of unique identifier (UI) specified in the program as Code.

ods produce acceptable allocations, and the actual choice depends on the availability of certain facilities for each method. Usually the randomization components of these two methods are produced by running a randomization software on either an Internet service provider or a local computer. The local encoder will obtain the next allocation (JIT method) or the entire random list (In Advance method) from the service provider or from the software on local computer and prepare the necessary blinded equipments.

Without the use of computer software or Internet services the maintenance of the whole process of randomization and allocation is difficult. In addition, in the case of any necessary restrictions on the process of randomization (i.e. block randomization [2]) the complexity of the process will be increased even further and be prone to errors. Randomization software may run on a local computer or may be hosted by an Internet server. A complete list of these software and services can be found on Martin Bland's web site [3]. Most of randomization software are hosted by websites for both JIT and In Advance methods, which require access to the Internet [4-8]. However, most of these Internet services have restricted capabilities with respect to the block design specification, control over the 
Table I: A simple randomized list produced by the software for a sample size of $\mathbf{3 0}$ subjects into three groups of Case, Control and Placebo with numeric sequential unique identifiers

\begin{tabular}{|c|c|c|c|}
\hline 00I: Case & 009: Placebo & 017: Control & 025: Placebo \\
\hline 002: Control & 010: Control & 018: Control & 026: Case \\
\hline 003: Case & 0II: Placebo & 019: Case & 027: Case \\
\hline 004: Case & 012: Control & 020: Control & 028: Placebo \\
\hline 005: Control & 013: Case & 02I: Placebo & 029: Placebo \\
\hline 006: Placebo & 014: Case & 022: Case & 030: Control \\
\hline 007: Placebo & 015: Placebo & 023: Case & \\
\hline 008: Control & 016: Control & 024: Placebo & \\
\hline
\end{tabular}

Table 2: The same setting as in table I, but with the numeric Uls in random order

\begin{tabular}{llll}
\hline 288: Case & 200: Control & 462: Placebo & 775: Case \\
644: Control & 437: Case & $448:$ Case & 622: Control \\
278: Placebo & 364: Control & 523: Control & 327: Control \\
427: Case & 525: Control & 837: Case & 514: Placebo \\
146: Placebo & 796: Case & 804: Placebo & 610: Case \\
383: Placebo & 208: Control & 581: Control & 167: Placebo \\
493: Placebo & 862: Placebo & 181: Control & \\
484: Case & 079: Case & 254: Placebo &
\end{tabular}

output format and flexibility of UIs. Among these available services, the tool in Randomization.com [4] seems to be more advanced than the others. It allows users to specify the number of subjects per block, the number of blocks and up to 20 treatment labels. Therefore, this service produces simple and block randomization using fixed and equal block sizes. Unfortunately, this service does not allow further restriction on block design (e.g., multiple block lengths or random variation in block number or size). The generated random list is in the form of UI and group name pairs, formatted in a single column, which in cases of large sample sizes may require further work to fit it in multiple columns for fine printing. Moreover, the block borders are not visible to allow for easy visual inspection of block sizes and equality of cases. Although a minor problem, Randomization.com only produces sequential numeric UIs with variable lengths (e.g. 1, 10, $100)$. The variability in lengths of UIs may disturb the visual impact of the generated list compared to the fixed length UIs (e.g. 001, 010, 100). Some researchers prefer to use random UIs in mixed alphanumeric format to decrease the likelihood of memorization and to improve the blindness of the study and concealment of allocations. Available randomization software have more restrictions in their capabilities than the Internet randomization services. They have limitations in their output format [9] and users can not specify the number and naming of the treatment groups $[10,11]$. In addition, these software are not designed with the capability to produce flexible UIs for participants. Therefore, the main objective of Random
Allocation Software was to construct a randomization software for parallel group trials with the following characteristics:

1. Independent running on a local computer without any need to access the Internet

2. Different types of program output: to file (html or text files), window and system clipboard

\section{Provisions for different block design}

4. Capability to deal with a larger number of groups

5. Specifying a name for each group

6. Control over the format, length and ordering of the generated UIs

7. Control over the format of generated sequence

8. Saving or loading the randomization settings

9. Viewing previously generated randomized sequences

\section{Implementation}

Random Allocation Software is a program created in Microsoft Visual Basic 6, and it installs in the same way as ordinary Windows software (i.e. running setup.exe and following on screen instructions). Once installed and run, 
there are some controls in the main window for specifying the number of groups (2 to 16), sample size and the name of each group. It also contains menu items to determine the program output and randomization settings. The default program output is saved into either html or text files, and it may also have output to a window or to the system clipboard. A variety of randomization options can be set in the options window. The length of generated UIs (named as Code in the program) can be between 3 to 10 characters and there are options for different alphanumeric structures. In addition, these UIs can appear in sequential or random order in the generated random list. The program can generate simple or block randomization in different types, including equal size blocks, multiple block lengths with random variation among the specified block sizes and complete randomized blocks (random number and size of blocks). The generated sequence will appear in a multiple columns format and the number of columns ( 1 to 10) can be changed in the options window. Output to html file will be formatted in the form of one block per table. Borders of the tables may be shown or hidden. By clicking the 'Generate' button in the main window the random sequence will be generated and opened by the default viewer for the output file (e.g. Internet Explorer for html files). Previously created output files can also be viewed from inside the program. Additional options include saving the current randomization settings, loading a previous setting and enabling the program to save the last setting upon program exit.

During execution, the program produces a random sequence of allocation using the Rnd function that generates a floating point random number. The Rnd function uses the linear-congruent method for pseudo-random number generation as depicted by the following formula:

$\mathrm{x} 1=\left(\mathrm{x} 00^{*} \mathrm{a}+\mathrm{c}\right) \operatorname{MOD}\left(2^{\wedge} 24\right)[12]$

where:

$\mathrm{x} 1$ = new value $\mathrm{x} 0=$ previous value (an initial value of 327680 is used by Visual Basic unless the Randomize X function is used to specify a different seed as $\mathrm{X}$ )

$\mathrm{a}=1140671485$

$C=12820163$

The seed of the Rnd will be the Timer function, which will return the number of seconds elapsed since midnight. Although this version of the program does not produce repeatable lists, it is possible to revise the program in subsequent versions to save the value of the seed to reproduce the same random list. The output consisted of shuffled allocations each of which is a UI, group name pair. The program checks for the uniqueness of the UIs and generates an error message if the specified UI length is insufficient to hold the entire sample size.

Runs test was used to check randomness of the output list with sample sizes from 10 to $190(10,30,50, \ldots, 190)$ and from 200 to $3000(200,600,1000, \ldots, 3000)$. Each runs test was carried out for the group number of 2, 3, 5 and 6 . SPSS 10 software was used to perform the runs test.

\section{Results}

The program starts running with the default settings. Users may run the program with the default settings or set the number of groups, the name of each group and the sample size. Clicking the 'Generate' button (figure 1) produces the random sequence. Before generating the random sequence, the option window will be displayed and different randomization settings can be entered (figure 2 and 3). Consider, for example, that we want to produce a simple randomized list for a sample size of 30 subjects into three groups of Case, Control and Placebo with numeric sequential UIs. After setting different options and clicking the 'Generate' button, the generated list will appear in columns (Table 1). Each entry in the list consists of a UI, and a group name pair. Alternatively numeric UIs may appear in random order (Table 2). Table 3 shows the output of the program for a block randomization with blocks of equal sizes.

Table 3: A block randomization list with four blocks of equal sizes

\begin{tabular}{|c|c|c|c|c|}
\hline 504: Placebo & 671: Placebo & 767: Case & 442: Control & 094: Placebo \\
\hline 256: Case & 636: Case & 200: Control & 677: Control & \\
\hline 669: Placebo & 355: Control & 334: Case & 765: Control & 073: Control \\
\hline 377: Case & 537: Placebo & 527: Placebo & 485: Case & \\
\hline 183: Placebo & 658: Control & 612: Case & 875: Control & 888: Case \\
\hline 733: Case & 127: Placebo & 864: Placebo & 476: Control & \\
\hline 552: Control & 138: Case & 548: Case & 938: Placebo & 592: Placebo \\
\hline 810: Control & 213: Control & 584: Placebo & 438: Case & \\
\hline
\end{tabular}




\begin{tabular}{|c|c|c|c|}
\hline 6396: Case & 2858: Control & 7146: Control & 6583: Case \\
\hline 4664: Control & 6168: Case & 2722: Case & 7919: Control \\
\hline 5281: Case & 4537: Case & 6032: Case & 2584: Control \\
\hline 0756: Control & 5054: Control & 7723: Case & 9227: Control \\
\hline 5076: Control & 0538: Control & 8239: Control & 7685: Case \\
\hline 5757: Case & 7352: Case & 3814: Control & 8112: Case \\
\hline 7374: Case & 6620: Control & 8124: Case & 3677 : Control \\
\hline 8997: Control & 4559: Case & 2261: Control & 1516: Control \\
\hline 8778: Control & 1273: Case & 6735: Control & 2133: Control \\
\hline 1205: Case & 9641: Case & 2145: Control & 6617: Case \\
\hline 5770: Control & 1167: Case & 2836: Case & 4331: Case \\
\hline 0180: Control & 5642: Control & 3353: Case & 3609: Case \\
\hline 7458: Case & 3375: Control & 5515: Control & \\
\hline 2387: Case & $1744:$ Case & 3248: Control & \\
\hline 4225: Case & 3562: Case & 8467: Control & 0236: Control \\
\hline 8791: Case & 4088: Case & 3042: Control & 1653: Control \\
\hline 3111: Control & 8663: Case & 1675: Case & 5237: Case \\
\hline 4892: Control & 9697: Control & 8143: Control & 1547: Case \\
\hline 5318: Case & 4362: Control & 0560: Control & 8815: Case \\
\hline 7471: Case & 8572: Control & 4134: Control & \\
\hline 4203: Case & 7841: Control & 9454: Case & \\
\hline 0432: Control & 1310: Control & 4711: Case & 5788: Case \\
\hline 4917: Case & 2292: Control & 6963: Case & 3157: Control \\
\hline 2631: Case & 3628: Control & 4695: Control & 5673: Case \\
\hline 1008: Control & 7283: Case & 2064: Control & 9258: Control \\
\hline 2525: Case & 2503: Case & 3581: Case & \\
\hline 6190: Case & 1861: Control & 7165: Control & \\
\hline
\end{tabular}

Figure 4

Sample output. Sample output for a block randomization with random block sizes.

Figure 4 is the printed output of the program for a block randomization with random block sizes.

In block randomization the final sample size is usually larger than the specified one.

A total of 18 runs test were performed to check the randomness of the program output, which resulted in $\mathrm{P}$ values of 0.22 to 0.81 .

\section{Discussion}

The main use of Random Allocation Software is to produce simple or block randomized sequences for parallel group trials. Its use is restricted to parallel group randomized trials. Compared with similar software, it enables the user to control the length, order and format of the UIs; and the type and format of the output. It allows specifying up to 16 groups for parallel trials. 


\section{Conclusions}

Random Allocation Software has been designed to produce random sequences consisting of UI, group name pairs with additional control over the output format and type. Available randomization software generally has limitations in the number of groups, naming each group, generating UIs and control over the output. Many of these problems have been addressed in the present software. As has been stated in previous sections, the main use of this software is for randomization in parallel group trials. The software can be revised to support crossover and other types of randomized trials. The experienced user may test the randomness of the program output by selecting numeric labels for group names and then exporting the generated list into a statistical software such as SPSS to execute a runs test on the exported data.

\section{Availability and requirements}

Project name: Random Allocation Software

Public use access:

http://www.msaghaei.com/Softwares/dnld/RA.zip (Latest version)

\section{http://ftp.mui.ac.ir/RA.zip}

Operating systems: Windows 98, Me, 2000, XP. It should be noted that on some Windows operating systems (especially Windows 2000) during installation of the program an error message like "Setup Cannot Continue... System Files Are Out of Date" may be displayed. If this happens, click OK and restart the system. Then run the setup.exe again. This is due to a known bug in the installation programs of Microsoft Visual Basic [13]. This problem has been removed from the newer versions of the program. Users are recommended to download the latest version from the first address.

Programming Language: Visual Basic 6

Other requirements: Internet Browser (Internet Explorer 5 or higher is recommended)

License: Free for academic use.

\section{Abbreviations}

JIT = Just in Time

$\mathrm{UI}=$ Unique Identifier

\section{Competing interests}

The author declares that he has no competing interests.

\section{Acknowledgements}

The author thanks Azra Kianinejad, Department of Human Development, Kobe University, Kobe, Japan for her kind review of the manuscript.

\section{References}

I. Schulz KF: Randomized controlled trials. Clin Obstet Gynecol 1998, 41:245-56.

2. Altman DG, Schulz KF, Moher D, Egger M, Davidoff F, Elbourne D, Gøtzsche PC, Lang T, CONSORT GROUP (Consolidated Standards of Reporting Trials): The Revised CONSORT Statement for Reporting Randomized Trials: Explanation and Elaboration. Ann of Intern Med 200I, I34:663-694.

3. Directory of randomization software and services [http:// www-users.york.ac.uk/ mb55/guide/randsery.htm]

4. Randomization.com [http://www.randomization.com/]

5. GraphPad QuickCalcs [http://www.graphpad.com/quickcalcs/ randomizel.cfm]

6. EDGAR [http://www.jic.bbsrc.ac.uk/services/statistics/edgar.htm]

7. PARADIGM Registration-Randomisation Software [http:// telescan.nki.nl/paradigm.html]

8. Randomizer [https://medinfo.uni-graz.at/randemo/web/about.php]

9. Simple Statistical Software by Martin Bland [http://wwwusers.york.ac.uk/ mb55/soft/soft.htm]

10. Randomization Generator download [http:// www.som.soton.ac.uk/staff/irg/files/Randomisation Generator download/]

II. KEYFINDER [http://lib.stat.cmu.edu/designs/]

12. Microsoft Knowledge Base Article - 231847 [http://sup port.microsoft.com/default.aspx?scid=kb;en-us;23|847]

13. Microsoft Knowledge Base Article - 174135 [http://sup port.microsoft.com/default.aspx?scid=kb;en-us; 174135\#appliesto]

\section{Pre-publication history}

The pre-publication history for this paper can be accessed here:

http://www.biomedcentral.com/1471-2288/4/26/prepub
Publish with Biomed Central and every scientist can read your work free of charge

"BioMed Central will be the most significant development for disseminating the results of biomedical research in our lifetime. " Sir Paul Nurse, Cancer Research UK

Your research papers will be:

- available free of charge to the entire biomedical community

- peer reviewed and published immediately upon acceptance

- cited in PubMed and archived on PubMed Central

- yours - you keep the copyright

Submit your manuscript here:

http://www.biomedcentral.com/info/publishing_adv.asp
BioMedcentral 